\title{
Neurotoxinas: perspectivas para a descoberta de novos analgésicos
}

\author{
Márcia Renata Mortari \\ Luzitano Brandão Ferreira²
}

\section{Resumo}

A dor é um fenômeno complexo, estando, muitas vezes, associada a condições limitantes. Muitas manifestações neuropáticas, as quais têm como sintoma sensação dolorosa intensa, continuam, ainda, sem tratamento. Em diversas outras patologias, quando há a disponibilidade, os efeitos colaterais constituem fatores de limitação para a terapia crônica. Vários estudos apontam para uma grande variedade de substratos neurais que podem ser alvos de intervenção farmacológica no controle da dor. Nesse contexto, as neurotoxinas derivadas de venenos de animais fornecem uma fonte vasta e, até certo ponto, inexplorada de compostos neuroativos. Dessa forma, o objetivo do presente trabalho foi o de descrever as neurotoxinas atualmente estudadas que podem ser utilizadas na prática clínica para o controle da dor.

Palavras-chave: Dor. Analgésicos. Neurotoxinas.

\section{Introdução}

Há milhões de anos, durante um contínuo processo evolutivo, vários animais invertebrados têm desenvolvido um conjunto de substâncias com atividade biológica nos seus venenos, que são usadas, principalmente, para paralisarem presas e/ou defender-se contra predadores.

Quando inoculados em seres humanos, esses venenos podem induzir reações alérgicas e diversas alterações do Sistema Nervoso Central (SNC), como sonolência, paralisia e convulsões. Essas substâncias que têm atividade sobre o SNC

\footnotetext{
${ }^{1}$ Bióloga, Doutora em Psicobiologia (USP). Professora da UnB, Distrito Federal, Brasil.

${ }^{2}$ Médico, Doutor em Genética (USP). Professor do UniCEUB e da Faculdade LS, Distrito Federal, Brasil. email: Luzitano-ferreira@uol.com.br.
} 
são conhecidas como neurotoxinas. Devido à alta afinidade e seletividade, várias dessas neurotoxinas vêm sendo estudadas para a produção de drogas destinadas ao tratamento de diversos distúrbios neurológicos, tais como epilepsia, doença de Parkinson, doença Alzheimer, bem como para o desenvolvimento de novas e mais eficazes drogas analgésicas.

\section{Neurofisiologia da dor}

A dor pode ser definida como uma sensação e experiência emocional desagradável, associada a um dano tissular real ou potencial (IASP, 1994). Aproximadamente, um terço da população mundial sofre de dor persistente ou recorrente, sendo essa uma queixa comum em pacientes portadores de diferentes doenças (ASHBURN; STAATS, 1999). Nesses casos, o tratamento é um desafio para pesquisadores e profissionais da saúde, que buscam, incessantemente, novas estratégias terapêuticas, uma vez que a maioria das já existentes é inadequada ou pode causar graves efeitos colaterais (STUCKY et al., 2001).

Analgésicos sistêmicos e terapias conservadoras são muito utilizados no controle da dor. Entretanto, em muitos casos, principalmente para os portadores de dor neuropática, são necessários tratamentos mais agressivos, os quais promovem uma melhora clínica significante em apenas 30 a $50 \%$ dos pacientes (SINDRUP; JENSEN, 1999; VILLETTI et al., 2003).

Diversos neurotransmissores e/ou neuromoduladores estão envolvidos nas várias etapas do processo da transmissão da nocicepção (MILLAN, 2002). Este é iniciado quando os nociceptores situados na pele, músculos ou órgãos viscerais são atingidos por estímulos mecânicos, térmicos, químicos ou elétricos. Essa estimulação gera potenciais de ação dependentes de sódio, que se propagam do final do nervo aferente primário até o corno dorsal da medula espinhal por meio de fibras mielinizadas e amielinizadas (MILLAN, 1999).

A chegada do potencial de ação aos terminais dos neurônios aferentes na medula espinhal leva à ativação de canais de cálcio voltagem-dependentes e ao influxo de $\mathrm{Ca}^{++}$, que culmina com a liberação de neurotransmissores e neuromoduladores, como a substância $\mathrm{P}$, o peptídeo relacionado à calcitonina (CGRP) e 
o L-glu (LEVINE, 1993; DICKENSON, 1997; BENNETT, 2000). Essas substâncias se ligam a receptores pós-sinápticos específicos e excitam ou sensibilizam os neurônios sensoriais secundários localizados na medula espinhal. Finalmente, esses subconjuntos de neurônios, projetam axônios para centros do cérebro, incluindo a formação reticular, tálamo e, por último, o córtex cerebral, no qual, é processada a informação nociceptiva (JULIUS; BASBAUM, 2001; McGIVERN, 2006).

Em relação à modulação do estímulo nociceptivo, as vias descendentes, que se originam no tronco cerebral e em outras estruturas cerebrais, apresentam um papel muito importante na modulação e integração dessas mensagens até o corno dorsal da medula espinhal. As vias serotonérgicas, noradrenérgicas e, em menor extensão, as dopaminérgicas compreendem os maiores componentes desse mecanismo descendente (MILLAN, 2002; FIELDS; BASBAUM, 1999). Em especial, sabe-se que a estimulação elétrica da Substância Cinzenta Periaquedutal (SCP) e do Núcleo Magno da Rafe (NMR) produz supressão da dor, sendo essas regiões denominadas Sistema Analgésico Central (SAC) (BASBAUM; FIELDS, 1984). O SAC está conectado com a formação reticular e o sistema límbico, recebendo colaterais do trato paleoespinotalâmico e enviando fibras eferentes serotoninérgicas que ativam neurônios encefalinérgicos inibitórios na substância gelatinosa da medula espinhal. Esse sistema analgésico pode ser ativado pelo estímulo doloroso, influenciar o processamento do sinal doloroso no nível do sistema límbico e regular a transmissão ao nível medular (MITCHELL et al., 2000; MILLAN, 2002).

Em decorrência das múltiplas mudanças neurofisiológicas que ocorrem na transmissão da informação nociceptiva, muitas estratégias têm sido desenvolvidas para induzir analgesia/antinocicepção, por exemplo: (1) antagonistas: para canais de $\mathrm{Ca}^{++}$(PRADO, 2001), canais de sódio voltagem-dependentes (SUZUKI; DICKENSON, 2000), receptores NMDA (SUZUKI et al., 2001); e (2) agonistas: GABAérgicos (MALCANGIO; BOWERY, 1996), colinérgicos (DECKER; MEYER, 1999), e de receptores para opióides (PLEUVRY; LAURETTI, 1996).

Nesse sentido, devido à grande especificidade e seletividade das neurotoxinas de animais, diversos estudos têm avaliado o perfil farmacológico desses compostos em modelos experimentais de indução de dor. Esses são potentes li- 
gantes de subtipos específicos de receptores ou canais iônicos e têm fornecido alternativas para a intervenção farmacológica com altos índices terapêuticos (RAJENDRA et al., 2004; MORTARI et al., 2007a).

\section{Neurotoxinas antinociceptivas}

Desde 1960, muitas neurotoxinas de artrópodes têm sido isoladas e caracterizadas. No SNC, essas moléculas podem atuar em diferentes alvos neuroniais, como: receptores, transportadores e canais iônicos em ambas as neurotransmissões excitatórias e inibitórias (BELEBONI et al., 2004; MELLOR; USHERWOOD, 2004). Sendo assim, essas neurotoxinas têm sido extremamente úteis tanto como ferramentas farmacológicas quanto na prospecção de novos fármacos para o tratamento de desordens neurológicas, como a epilepsia e a isquemia, e para o controle da dor (SHEN et al., 2000; WANG; CHIN, 2004).

Com relação às neurotoxinas com atividade antinociceptiva, Roerig e Howse (1996) relataram o efeito da $\omega$-agatoxina IVA ( $\omega$-Aga-IVA), isolada do veneno da aranha Agelenopsis aperta, contra estímulo térmico no teste da retirada de cauda (tail-flick), quando co-administrado por via intratecal com morfina e clonidina. O uso desse peptídeo como analgésico poderia trazer benefícios, particularmente, para pacientes tolerantes ou dependentes de opióides, uma vez que esse composto apresenta seletividade para canais para íons $\mathrm{Ca}^{++}$ do tipo P/Q (RAJENDRA et al, 2004).

Estudos têm descrito que a administração intratecal de bloqueadores não-seletivos de canais de íons $\mathrm{Ca}^{++}$apresenta efeito antinociceptivo em animais submetidos a testes com estímulos térmicos: placa quente (hot-plate) e retirada de cauda (tail-flick) (REDDY; YAKSH, 1980). Segundo Malmberg \& Yaksh (1994), canais de íons $\mathrm{Ca}^{++}$dos tipos $\mathrm{N}$ e P estão envolvidos em comportamentos nociceptivos induzidos pela injeção de formalina em ratos, enquanto os canais do tipo L não apresentam efeito. Sendo assim, bloqueadores de canais de íons de $\mathrm{Ca}^{++}$do tipo P podem apresentar uma predominante importância no controle da excitação dos neurônios espinhais, a partir de aferências sensoriais de tecidos inflamados, aliviando a dor inflamatória (NEBE et al., 1997). 
Além dos peptídeos, alguns estudos avaliaram a atividade das poliaminas Jorotoxina e Philantotoxina e o papel funcional de receptores AMPA/Cainato permeáveis ao $\mathrm{Ca}^{++}$no processamento nociceptivo da dor (KAWAI et al, 1991). Observou-se que a administração intratecal de diferentes doses dessas toxinas bloqueou a alodínia induzida termicamente (SORKIN et al., 1999) e a hiperalgesia (STANFA et al., 2000). O efeito dessas neurotoxinas pode sugerir um possível envolvimento de receptores AMPA localizados em interneurônios GABAérgicos medulares e dos receptores cainato, ambos permeáveis ao $\mathrm{Ca}^{++}$na estimulação excitatória espinhal durante o processo nociceptivo. Em contraste, as respostas evocadas por receptores NMDA foram consideradas insignificantes (JONES; LODGE, 1991; SORKIN et al., 2001).

Uma nova classe de toxinas peptídicas (Psalmotoxina 1; PcTx1) foi isolada da peçonha da aranha neotropical Psalmopoeus cambridgei. Em princípio, a PcTx1 bloqueia canais iônicos sensíveis a ácido (ASIC) ou canais catiônicos ativados por $\mathrm{H}^{+}$(ESCOUBAS et al., 2000). Esses canais apresentam um papel importante em condições patológicas, como isquemia cerebral ou epilepsia, sendo, também, responsáveis pela sensação de dor que acompanha a acidose tecidual e a inflamação (McCLESKEY; GOLD, 1999). Uma vez que a acidificação externa é um dos principais fatores de dor associada à inflamação (hematose, isquemia cardíaca ou muscular e câncer), essas neurotoxinas podem ser utilizadas no controle da recepção da dor desencadeada por esses canais (WALDMANN et al., 1999).

Recentemente, foi isolado um composto com atividade antinociceptiva do veneno da vespa social brasileira Polybia occidentalis. O peptídeo isolado e identificado pertence à classe das neurocininas e já foi descrito anteriormente, sendo denominado Thr'-Bradicinina (MORTARI et al., 2006b). A primeira descrição dessa molécula em vespas deu-se na peçonha da vespa social Polistes rothneyi iwatai, há 30 anos (WATANABE et al, 1976), e, mais tarde, no veneno da vespa solitária Megascolia flavifrons (YASUHARA et al., 1987). No entanto, a descrição foi inédita no gênero Polybia.

A neurocinina $T h r^{6}-B K$ é um pequeno peptídeo formado por nove resíduos de aminoácidos, Arg-Pro-Pro-Gly-Phe-Thr-Pro-Phe-Arg-OH, o qual apresenta uma alta homologia com a bradicinina (BK), com exceção da substituição da Ser na BK por Thr na Thr'-BK na posição seis, e, em decorrência disso, há pe- 
quenas modificações em suas estruturas secundárias (PELLEGRINI et al., 1997). Essa modificação tem sido indicada como responsável pelo aumento da afinidade pelo receptor $\mathrm{B}_{2}$ e da potência da $T h r^{6}-\mathrm{BK}$ em relação à $\mathrm{BK}$, em investigações in vitro (PELLEGRINI et al., 1997; PELLEGRINI; MIERKE, 1997).

Nessa perspectiva, Mortari e colaboradores (2007b) demonstraram que a Thr ${ }^{6}$-BK apresenta efeito antinociceptivo dependente de dose e de tempo, quando injetada diretamente no SNC de ratos nos testes: hot-plate e tail-flick, sendo que a Thr ${ }^{6}$-BK se mostrou 3 vezes mais potente que a morfina e 4 vezes mais potente que a BK no teste do tail-flick. Segundo os autores, a Thr ${ }^{6}$-BK induz antinocicepção por meio da ativação de receptores do tipo $\mathrm{B}_{2}$ pré-sinápticos, os quais ativam vias descendentes adrenérgicas. Os aspectos funcionais desse trabalho foram evidenciados por Nagy e colaboradores (2007), os quais afirmam que o trabalho de Mortari e colegas (2007) e os estudos que investigam o papel das cininas sobre o SNC fornecem novas informações sobre o sistema supra-espinhal, cuja modulação pode representar uma nova estratégia no controle de patologias relacionadas à dor (NAGY et al., 2007). Além de induzir antinocicepção, o veneno de $P$. occidentalis possui componentes anticonvulsivantes. Estes protegeram ratos Wistar de crises evocadas pelos convulsivantes químicos picrotoxina, ácido caínico e PTZ, sem induzir toxicidade motora na dose efetiva (MORTARI et al., 2005).

Outros estudos relacionam a peçonha da aranha $P$. bistriata, uma aranha colonial que se distribui pelos cerrados da América do Sul, com atividades analgésicas. A caracterização dos componentes do veneno dessa aranha começou quando foi observado que a injeção do veneno bruto em térmitas provoca paralisia desses isópteros (FONTANA et al., 2000). A ação paralisante do veneno é essencial para sobrevivência da aranha. Ela paralisa suas presas, mantendo-as vivas até serem embalsamadas e, posteriormente, utilizadas na sua alimentação (USHERWOOD; BLAGBROUGH, 1991). Rodrigues e colaboradores (2001) mostraram que o veneno da $P$. bistriata é heterogêneo e possui componentes com atividades farmacológicas diversificadas. Esses autores demonstraram que a injeção por via intracerebroventricular (icv) do veneno bruto de P. bistriata causa crises límbicas, enquanto o veneno desproteinizado bloqueia crises generalizadas tônico-clônicas, induzidas pelos antagonistas gabaérgicos bicuculina, 
picrotoxina e pentilenotetrazol (PTZ), quando injetados por via intracerebroventricular (i.c.v.) em ratos Wistar, por alterar as captações de GABA e L-Glu (CAIRRÃO et al., 2002).

Diversas toxinas do veneno de P. bistriata foram identificadas, como a inosina, que apresenta efeito paralisante sobre insetos e pró-convulsivante em ratos (RODRIGUES et al., 2004a), e o composto Parawixina I, o qual estimula a recaptação de glutamato em sinaptossomas cérebro corticais de ratos, além de atuar como neuroprotetor em modelo de lesão da retina de ratos (FONTANA et al., 2003). Um mecanismo de ação inédito para compostos isolados de veneno foi descrito para a Parawixina I, a qual aumenta seletivamente o influxo de L-Glu por transportadores de EET2 em liposomas reconstituídos e células COS (FONTANA et al., 2007). Segundo Torres-Salazar \& Fahlke (2007), a seletividade e especificidade da Parawixina I fazem com que essa molécula possa ser considerada como um ponto de partida para síntese de novas drogas a serem utilizadas como terapia em patologias que envolvem alterações no sistema glutamatérgico.

Outro composto isolado, a FrPbA2, apresenta, seletivamente, um potente efeito inibitório sobre a recaptação do GABA e da glicina, além de ser anticonvulsivante contra crises agudas induzidas pela bicuculina (CAIRRÃO et al., 2002), picrotoxina, pilocarpina, ácido caínico e PTZ, sem induzir toxicidade motora na dose efetiva (GELFUSO et al., 2007). Mais recentemente, a estrutura química desse composto foi elucidada juntamente com parte do seu mecanismo de ação. Tratase de um composto pequeno, de estrutura análoga à do GABA, sendo que esta molécula não altera a atividade dos canais de $\mathrm{Na}^{+}, \mathrm{K}^{+}$e $\mathrm{Ca}^{++}$nem possui efeito sobre receptores de GABA, sobre a GABA transaminase ou sobre transporte reverso (BELEBONI et al., 2006). Apesar de encorajadores, os resultados obtidos com o estudo dos compostos de baixo peso molecular do veneno da aranha P. bistriata são preliminares. Apenas uma pequena parte desses compostos foi avaliada até agora.

\section{Considerações Finais}

O uso de toxinas de animais como ferramentas em neurociências tem contribuído para elucidação de aspectos funcionais do Sistema Nervoso Central. Apesar da imensa quantidade de animais venenosos e suas toxinas, especialmente no 
Brasil, país com a maior biodiversidade do planeta, o potencial terapêutico dos venenos é modestamente estudado e utilizado. Faz-se necessário investigar o potencial terapêutico dos compostos presentes em diferentes peçonhas de animais, no intuito de fornecer novas ferramentas para investigação de processos patológicos e para síntese de novos compostos com ação terapêutica para o controle da dor.

\section{Neurotoxins: prospects for the discovery of new painkillers}

\section{Abstract}

Pain is a complex phenomenon, commonly associated to limiting conditions. Several neuropathies share symptoms of intense pain, which is often untreated and when treatment is available, it is always followed by strong untoward side effects, limiting chronic administration. Research focusing pain has pointed to a wide variety of neuronal substrates that function as targets to pharmacological intervention in the control of pain states. In this context, toxins from animal venoms provide a vast and yet unexplored source of neuroactive compounds, which selectively activates or inhibits important mammalian neuronal structures. Thus, the purpose of this work was to describe the neurotoxins currently studied that can be used in clinical practice for the control of pain.

Keywords: Pain. Antinociception. Neurotoxins.

\section{Referências}

ASHBURN, M. A.; STAATS, P. S. Management of chronic pain. Lancet, [S.L], v. 353, n. 29 , p. $1865-1869,1999$.

BASBAUM, A. I.; FIELDS, H. L. Endogenous pain control systems: brainstem spinal pathways and endorphin circuitry. Annual Review of Neuroscience, [S.L], v. 7, p. 309-38, 1986.

BELEBONI R. O. et al. Neurochemical characterization of a neuroprotective compound from Parawixia bistriata spider venom that inhibits synaptosomal uptake of GABA and glycine. Molecular Pharmacology, v. 69, n. 6, p. 1998-2006, 2006. 
BELEBONI, R. O. et al. Spider and wasp neurotoxins: pharmacological and biochemical aspects. European Journal of Pharmacology, [S.L], v. 493, p. 1-17, 2004.

BENNETT, G. J. Introduction to special review series: experimental advances in understanding cancer pain. Pain Medicine, [S.L], v. 1, n. 4, p. 295, 2000.

CAIRRÃO, M. A. R. et al. Anticonvulsant and GABA uptake inhibition properties of $P$. bistriata and S. raptoria spider venom fractions. Pharmacology \& Biology, [S.L], v. 40, p. 472-477, 2002.

DECKER, M. W.; MEYER, M. D. Therapeutic potential of neuronal nicotinic acetylcholine receptor agonists as novel analgesics. Biochemistry Pharmacology, [S.L], v. 58, n. 6, p. 917-23, 1999.

DICKENSON, A. H. Plasticity: implications for opioid and other pharmacological interventions in specific pain states. Behaviour Brain Science, [S.L], v. 20, n. 3, p. 392-403, 1997.

ESCOUBAS, P. et al. Isolation of a tarantula toxin specific for a class of protongated $\mathrm{Na}^{+}$channels. Journal of Biology Chemistry, [S.L], v. 275, n. 33, p. 25116$21,2000$.

FIELDS, H. L.; BASBAUM, A. I. Central nervous system mechanism of pain modulation. In: WALL, P. D.; MELZACK, R.Textbook of pain. Ediburgh: Churchill Livingstone, 1999.

FONTANA A. C. K. et al. Paralizing activity of the Parawixia bistriata crude venom in termites: a new bioassay. Toxicon, [S.L], v. 38, p. 133-138, 2000.

FONTANA, A. C. Purification of a neuroprotective component of Parawixia bistriata spider venom that enhances glutamate uptake. British Journal of Pharmacology, [S.L], v. 139, n. 7, p. 1297-309, 2003.

FONTANA A. C. et al. Enhancing glutamate transport: mechanism of action of Parawixin1, a neuroprotective compound from Parawixia bistriata spider venom. Molecular Pharmacology, [S.L], v. 72, n. 5, p. 1228-37, 2007.

GELFUSO E. A. et al. Neuropharmacological profile of FrPbAII, purified from the venom of the social spider Parawixia bistriata (Araneae, Araneidae). Life Sciences, [S.L], v. 80, n. 6, p. 566-72, 2007. 
MERSKEY; BOGDUK. N. Classification of Chronic Pain. 2. ed. Seattle: IASP Press, 1994.

JONES, M. G.; LODGE, D. Comparison of some arthropod toxins and toxin fragments as antagonists of excitatory amino acid-induced excitation of rat spinal neurones. European Journal of Pharmacology, [S.L], v. 204, n. 2, p. 203-209, 1991.

JULIUS, D.; BASBAUM, A. I. Molecular mechanisms of nociception. Nature, [S.L], v. 413, n. 6852, p. 203-210, 2001.

KAWAI, N. et al. Spider toxin and the glutamate receptors. Comparative Biochemistry and Physiology, [S.L], v. 98, n. 1, p. 87-95, 1991.

LEVINE, J. D. et al. Peptides and the primary afferent nociceptor. Journal of Neurosciences, [S.L], v. 13, n. 6, p. 2273-2286, 1993.

MALCANGIO, M.; BOWERY, N. G. GABA and its receptors in the spinal cord. Trends in Pharmacology Sciences, [S.L], v. 17, n. 12, p. 457-462, 1996.

MALMBERG, A. B.; YAKSH, T. L. Antinociception produced by spinal delivery of the $\mathrm{S}$ and $\mathrm{R}$ enantiomers of flurbiprofen in the formalin test. European Journal of Pharmacology, [S.L], v. 256, n. 2, p. 205-209, 1994.

MCCLESKEY, E. W.; GOLD, M. S. Ion channels of nociception. Annual Review of Physiology, [S.L], v. 61, p. 835-56, 1999.

MCGIVERN, J. G. Targeting N-type and T-type calcium channels for the treatment of pain. Drug Discovery Today, [S.L], v. 11, n. 5, p. 245-53, 2006.

MELLOR, I. R.; Usherwood, P. N. R. Targeting ionotropic receptors with polyaminecontaining toxins. Toxicon, [S.L], v. 43, p. 493-508, 2004.

MILLAN, M. J. Descending control of pain. Progress in Neurobiol, [S.L], v. 66, n. 6, p. 355-474, 2002.

MILLAN, M. J. The induction of pain: an integrative review. Progress in Neurobiology, [S.L], v. 57, n. 1, p. 1-164, 1999.

MITCHELL, J. M. et al. A locus and mechanism of action for associative morphine tolerance. Nature Neuroscieces, [S.L], v. 3, n. 1, p. 47-53, 2000. 
MORTARI, M. R. et al. Anticonvulsant and behavioural effects of the denatured venom of the social wasp Polybia occidentalis (Polistinae, Vespidae). Basic Clinical Pharmacology and Toxicology, [S.L], v. 97, n. 5, p. 289-95, 2006.

MORTARI, M. R. et al. Neurotoxins from invertebrates as anticonvulsants: from basic research to therapeutic application. Pharmacology \& Therapeutics, [S.L], v. 114 , p. 171-83, 2007a.

MORTARI M. R. et al. Inhibition of acute nociceptive responses in rats after i.c.v. injection of Thr6-bradykinin, isolated from the venom of the social wasp, Polybia occidentalis. British Journal of Pharmacology, [S.L], v. 151, p. 860-869, 2007 b.

NAGY I. et al. Taking the sting out of pain. British Journal of Pharmacology, [S.L], v. 151, n. 6, p. 721-722, 2007.

NEBE, J. et al. Omega-agatoxin IVA, a P-type calcium channel antagonist, reduces nociceptive processing in spinal cord neurons with input from the inflamed but not from the normal knee joint-an electrophysiological study in the rat in vivo. European Journal of Neurosciences, [S.L], v. 9, n. 10, p. 2193-2201, 1997.

PELLEGRINI, M. et al. Threonine-bradykinin: structural characterization in the presence of micelles by nuclear magnetic resonance and distance geometry. Journal of Medical Chemistry, [S.L], v. 40, p. 92-98, 1997.

PELLEGRINI, M.; MIERKE, D. F. Threonine6-Bradykinin: molecular dynamics simulations in abiphasic membrane mimetic. Journal of Medical Chemistry, [S.L], v. 40, p. 99-104, 1997.

PLEUVRY, B. J.; LAURETTI, G. R. Biochemical aspects of chronic pain and its relationship to treatment. Pharmacology \& Therapeutics, [S.L], v. 71, n. 3, p. 313324, 1996.

PRADO, W. A. Involvement of calcium in pain and antinociception. Brazilian Journal of Medical and Biological Research, [S.L], v. 34, n. 4, p. 449-461, 2001.

RAJENDRA, W. et al. Toxins in anti-nociception and anti-inflammation. Toxicon, [S.L], v. 44, n. 1, p. 1-17, 2004.

REDDY, S. V.; YAKSH, T. L. Antinociceptive effects of lanthanum neodymium and europium following intrathecal administration. Neuropharmacology, [S.L], v. 19, n. 2, p. 181-185, 1980. 
RODRIGUES, M. C. et al. The biological activity in mammals and insects of the nucleosidic fraction from the spider Parawixia bistriata. Toxicon, [S.L], v. 43, p. 375-383, 2004.

ROERIG, S. C.; HOWSE, K. M. Omega-agatoxin IVA blocks spinal morphine/ clonidine antinociceptive synergism. European Journal of Pharmacology, [S.L], v. 314, n. 3, p. 293-300, 1996.

SHEN, G. S. et al. Conopeptides: From deadly venoms to novel therapeutics. Drug Discovery Today, [S.L], v. 5, p. 98-106, 2000.

SINDRUP, S. H.; JENSEN, T. S. Efficacy of pharmacological treatments of neuropathic pain: an update and effect related to mechanism of drug action. Pain, [S.L], v. 83, n. 3, p. 389-400, 1999.

SORKIN, L. S. et al. Mechanical allodynia in rats is blocked by a $\mathrm{Ca}^{2+}$ permeable AMPA receptor antagonist. Neuroreport, [S.L], v. 10, n. 17, p. 3523-3526, 1999.

STANFA, L. C. et al. Role of $\mathrm{Ca}^{2+}$-permeable non-NMDA glutamate receptors in spinal nociceptive transmission. Neuroreport, [S.L], v. 11, n. 14, p. 3199-202, 2000.

STUCKY, C. L. et al. Mechanisms of pain. Prococeedings of the National Academy of Sciences USA, [S.L], v. 98, n. 21, p. 11845-1146, 2001.

SUZUKI, R.; DICKENSON, A. H. Neuropathic pain: nerves bursting with excitement. Neuroreport, [S.L], v. 12, n. 11, p. R17-21, 2000.

SUZUKI, R. et al. Comparison of the effects of MK-801, ketamine and memantine on responses of spinal dorsal horn neurones in a rat model of mononeuropathy. Pain, [S.L], v. 91, n. 2, p. 101-109, 2001.

TORRES-SALAZAR, D.; FAHLKE, C. Parawixin1: a spider toxin opening new avenues for glutamate transporter pharmacology. Molecular Pharmacology, [S.L], v. 72, n. 5, p. 1100-1102, 2007.

USHERWOOD, P. N. R.; BLAGBROUGH, I. S. Spider toxins affecting glutamate receptors: polyamines in therapeutic neurochemistry. Phamacology \& Therapeutics, [S.L], v. 52, p. 245-268, 1991.

VILLETTI, G. et al. Preclinical evaluation of CHF3381 as a novel antiepileptic agent. Neuropharmacology, [S.L], v. 40, p. 866-878, 2001. 
WALDMANN, R. et al. H(+)-gated cation channels. Annual New York Acadademy Sciences, [S.L], v. 868, p. 67-76, 1999.

WANG, C. Z.; CHIN, C. W. Conus peptides: a rich pharmaceutical treasure. Acta Biochimica Biophysica, [S.L], v. 36, n. 11, p. 713-23, 2004.

WATANABE, M., Occurrence of thr-6-bradykinin and its analogous peptide in the venom of Polistes rothmeyi iwatai. Animal, Plant and Microbial Toxins, [S.L], v. 2, p. 105-112, 1976.

YASUHARA, T. et al. Two kinins isolated from an extract of the venom reservoirs of the solitary wasp Megascolia flavifrons. Toxicon, [S.L], v. 25, p. 527-535, 1987. 